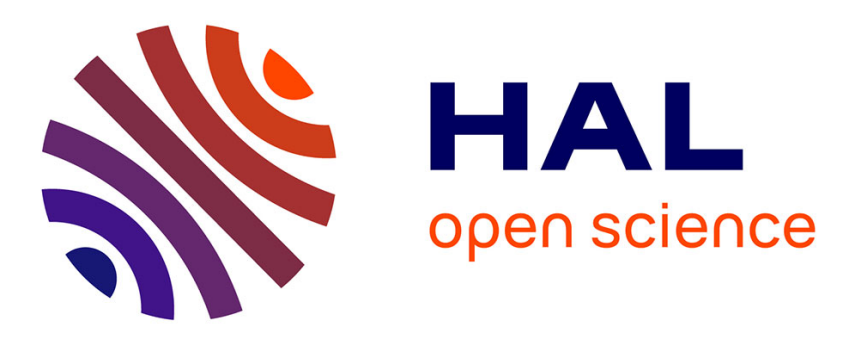

\title{
The Impact of Variation Operators on the Performance of SMS-EMOA on the Bi-objective BBOB-2016 Test Suite
}

Anne Auger, Dimo Brockhoff, Nikolaus Hansen, Dejan Tušar, Tea Tušar, Tobias Wagner

\section{To cite this version:}

Anne Auger, Dimo Brockhoff, Nikolaus Hansen, Dejan Tušar, Tea Tušar, et al.. The Impact of Variation Operators on the Performance of SMS-EMOA on the Bi-objective BBOB-2016 Test Suite. GECCO 2016 - Genetic and Evolutionary Computation Conference, Jul 2016, Denver, CO, United States. pp.1225 - 1232, 10.1145/2908961.2931705 . hal-01435456

\author{
HAL Id: hal-01435456 \\ https://hal.inria.fr/hal-01435456
}

Submitted on 14 Jan 2017

HAL is a multi-disciplinary open access archive for the deposit and dissemination of scientific research documents, whether they are published or not. The documents may come from teaching and research institutions in France or abroad, or from public or private research centers.
L'archive ouverte pluridisciplinaire HAL, est destinée au dépôt et à la diffusion de documents scientifiques de niveau recherche, publiés ou non, émanant des établissements d'enseignement et de recherche français ou étrangers, des laboratoires publics ou privés. 


\section{The Impact of Variation Operators on the Performance of SMS-EMOA on the Bi-objective BBOB-2016 Test Suite}

\author{
Anne Auger* \\ Dejan Tušar• \\ ${ }^{\star}$ Inria Saclay-lle-de-France \\ TAO team, France \\ LRI, Univ. Paris-Sud \\ firstname.lastname@inria.fr
}

\author{
Dimo Brockhoff• \\ Tea Tušar• \\ -Inria Lille Nord-Europe \\ DOLPHIN team, France \\ Univ. Lille, CNRS, UMR 9189 - CRIStAL \\ firstname.lastname@inria.fr
}

\author{
Nikolaus Hansen* \\ Tobias Wagner ${ }^{\diamond}$ \\ ${ }^{\diamond}$ TU Dortmund University \\ Institute of Machining \\ Technology (ISF), Germany \\ wagner@isf.de
}

\begin{abstract}
The S-metric-Selection Evolutionary Multi-objective Optimization Algorithm (SMS-EMOA) is one of the best-known indicator-based multi-objective optimization algorithms. It employs the S-metric or hypervolume indicator in its (steadystate) selection by deleting in each iteration the solution that has the smallest contribution to the hypervolume indicator. In the SMS-EMOA, the conceptual idea is this hypervolumebased selection. Hence the algorithm can, for example, be combined with several variation operators. Here, we benchmark two versions of SMS-EMOA which employ differential evolution (DE) and simulated binary crossover (SBX) with polynomial mutation (PM) respectively on the newly introduced bi-objective bbob-biobj test suite of the Comparing Continuous Optimizers (COCO) platform. The results unsurprisingly reveal that the choice of the variation operator is crucial for performance with a clear advantage of the DE variant on almost all functions.
\end{abstract}

\section{Keywords}

Benchmarking, Black-box optimization, Bi-objective optimization

\section{INTRODUCTION}

Indicator-based algorithms constitute an important class of (stochastic) multi-objective optimization algorithms. The S-metric-Selection Evolutionary Multi-objective Optimization Algorithm (SMS-EMOA) [4] is among the best known multi-objective algorithms and as such rather an algorithm framework with a fixed environmental selection. It uses the so-called S-metric or hypervolume contribution or hypervolume indicator loss to assign a quality to each solution in its selection procedure but does not specify the variation operators used to create new candidate solutions. Note that the hypervolume loss selection criterion is, in more general forms, also used in other well-known algorithms such as

(C) The authors, 2016. This is the authors' version of the work. It is posted here by permission of ACM for your personal use. Not for redistribution. The definitive version was published at GECCO'16, July 20-24, 2016, Denver, CO, USA, http://dx.doi.org/10.1145/2908961.2931705 the MO-CMA-ES [12] or HypE [3]. It is therefore natural to benchmark an algorithm with hypervolume-based selection on the recently proposed bi-objective bbob-biobj test suite [15] of the Comparing Continuous Optimizers platform (COCO, [10]) and to investigate the impact of different variation operators on the algorithm's performance.

\section{ALGORITHM PRESENTATION}

The main functionality of the SMS-EMOA is summarized in the pseudo code of Algorithm 1. After a random population initialization, the algorithm performs iteration-wise the following steps until the total budget is exhausted: First, a single new candidate solution is created from the current population via a to-be-specified variation operator and evaluated on the vector-valued objective function. Then, a non-dominated ranking [8] is performed on the population augmented by the new point. Within the solutions of worst rank, the hypervolume loss of each solution to this set is computed, i.e. the amount of hypervolume difference between the set of worst rank with and without this solution. Finally, the solution with the smallest hypervolume loss is deleted ${ }^{1}$ and the next iteration starts. The hypervolume loss computation depends on the reference point of the hypervolume indicator, which is typically chosen relative to the current population in the SMS-EMOA. This makes its hypervolumebased selection unique from others with fixed reference point such as the ones in the MO-CMA-ES or HypE.

\subsection{Algorithm Variants}

In the following, we compare two SMS-EMOA variants that differ only in the variation operators used. On the one hand, we have SMS-EMOA with polynomial mutation (PM, [7]) and simulated binary crossover (SBX, [6]): we denote this variant by SMS-PM and abbreviate it further in the tables to PM. On the other hand, we have an SMS-EMOA variant that employs differential evolution [14] and that we call SMS-DE or DE for short. In addition, we display the performance of a pure random search within $[-5,5]^{n}[1]$ as a baseline.

\footnotetext{
${ }^{1}$ In practice, the computation of the hypervolume loss is skipped if only one solution with worst rank exists in the population.
} 


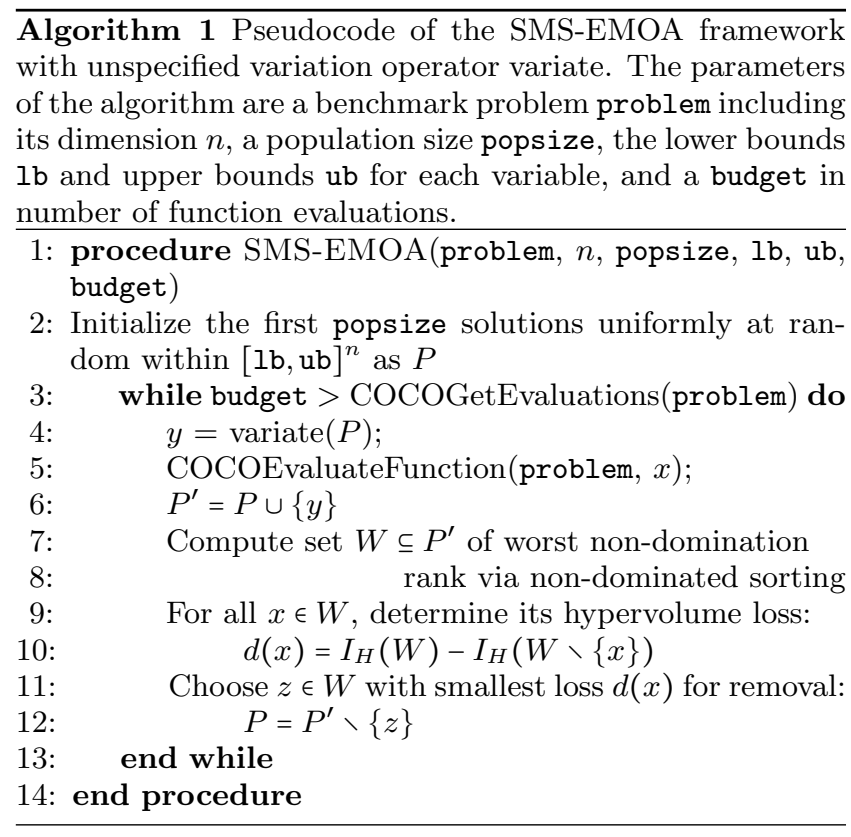

\subsection{Parameters}

The two benchmarked variants of the SMS-EMOA use a fixed population size of 100 and a dynamic reference point $r=\left(r_{1}, r_{2}\right)$ for the hypervolume computation defined by $r_{i}=\max _{p \in W} f_{i}(p)+1(i \in\{1,2\})$ where $W$ is the current set of solutions with worst non-domination rank. The variant using simulated binary crossover and polynomial mutation applies the parameters $\eta_{c}=15$ and $\eta_{m}=20$. The crossover probability is set to 0.9 , the swap probability to 0.5 , and the mutation probability per component to $1 / n$, where $n$ denotes the problem's input dimension. The DE variant applies the simple scheme, where one difference of two random solutions is added to a third one. The weight of the difference is set to $F=0.2+0.6 R$ with $R \in[0,1]$ being sampled from a uniform random distribution. The crossover probability is set to $C R=0.9$. Starting from a random index, only one block is used for the crossover. The length of the block is determined by sampling $R \in[0,1]$ from a uniform random distribution until $R>C R$. No restarts are implemented.

\section{CPU TIMING}

In order to evaluate the CPU timing of the algorithm, we have run the two SMS-EMOA variants SMS-PM and SMS$\mathrm{DE}$, on the entire bbob-biobj test suite for $500 \cdot n$ function evaluations, i.e. for $5 \cdot n$ generations and a population size of 100 . The Matlab code was run under Matlab 2008b on a Windows XP machine with Intel(R) Core(TM)2 Duo T9600 CPU $2.80 \mathrm{GHz}$ with 1 processor and 2 cores. The time per function evaluation over different dimensions is shown in Table 1. We observe that the dimension has almost no effect on the time per function evaluation for the algorithms tested and the relatively low budget of $500 \cdot n$ function evaluations. We also see slightly smaller times per function evaluation for the SMS-EMOA variant employing polynomial mutation.

\section{RESULTS AND DISCUSSIONS}

Results from experiments according to [11], [9] and [5] on the benchmark functions given in [15] are presented in
Table 1: Results of CPU timing experiment in runtime per function evaluation (in $10^{-4}$ seconds) for the two SMS-EMOA variants, benchmarked here.

\begin{tabular}{ccccccc} 
& \multicolumn{6}{c}{ time per function evaluation (in $\left.10^{-4} \mathrm{~s}\right)$} \\
algorithm & $2-\mathrm{D}$ & $3-\mathrm{D}$ & $5-\mathrm{D}$ & $10-\mathrm{D}$ & $20-\mathrm{D}$ & $40-\mathrm{D}$ \\
\hline SMS-PM & 5.8 & 5.8 & 5.6 & 5.6 & 5.6 & 5.7 \\
SMS-DE & 6.2 & 6.1 & 6.2 & 5.8 & 5.7 & 5.9
\end{tabular}

Figures 1, 2, 3 and 4 and in Tables 2 and 3. The experiments were performed with COCO [10], version 1.0.1, the plots were produced with version 1.1.1.

The average running time (aRT), used in the figures and tables, depends on a given quality indicator value, $I_{\text {target }}=I^{\text {ref }}+\Delta I$, and is computed over all relevant trials as the number of function evaluations executed during each trial while the best indicator value did not reach $I_{\text {target }}$, summed over all trials and divided by the number of trials that actually reached $I_{\text {target }}[11,13]$. Statistical significance is tested with the rank-sum test for a given target $I_{\text {target }}$ using, for each trial, either the number of needed function evaluations to reach $I_{\text {target }}$ (inverted and multiplied by $-1)$, or, if the target was not reached, the best $\Delta I$-value achieved, measured only up to the smallest number of overall function evaluations for any unsuccessful trial under consideration.

Investigating the performance data in the shown figures and tables, results in the following general observations.

In an initial phase, both tested SMS-EMOA variants show a performance worse than the pure random search without reaching any (or just a few) hypervolume indicator targets in the first $100 n$ function evaluations. This can be attributed to the fact that the pure random search is sampling within $[-5,5]^{n}$ while the initial population of the SMS-EMOA variants is chosen uniformly at random within the much larger space $[-100,100]^{n}$, see also [2], and thus their populations need more time to approach the Pareto set.

After this initial phase, SMS-PM is almost always better than SMS-DE in the beginning with the Attractive Sector/Different Powers function (f23) being the only exception in 10-D. The difference, however, is relatively small with function $\mathrm{f} 12$ (in 10-D) and the separable-moderate function group (in 5-D) showing the largest effects of a factor of around 2 .

Slightly after this, SMS-DE takes over and is better in almost all functions with respect to the number of solved targets at the end of the run. The only exceptions in 10D are the Sphere/sep. Ellipsoid function (f2) where SMSPM stays better all the time, the sep. Ellipsoid/sep. Elliposid function (f11) where SMS-PM is taking over again around $4 . .4 .5 \cdot 10^{5} n$ function evaluations, and the sep. Ellipsoid/Sharp ridge (f14) on which both algos have equal performance after $10^{3} n$ function evaluations. But also here, the difference between the algorithms is relatively small: only on the Sphere/Sphere (f1), the Sphere/Different Powers (f6), and the Attractive sector/Attractive sector (f20) functions are the proportions of solved target precisions in 10-D larger than $20 \%$ for some budgets.

With respect to the baseline random search, the SMSEMOA variants are better for budgets larger than around $10^{3} n$ on most functions, except for Sphere/sep. Ellipsoid (f2) and sep. Ellipsoid/sep. Ellipsoid (f11). But also for other 

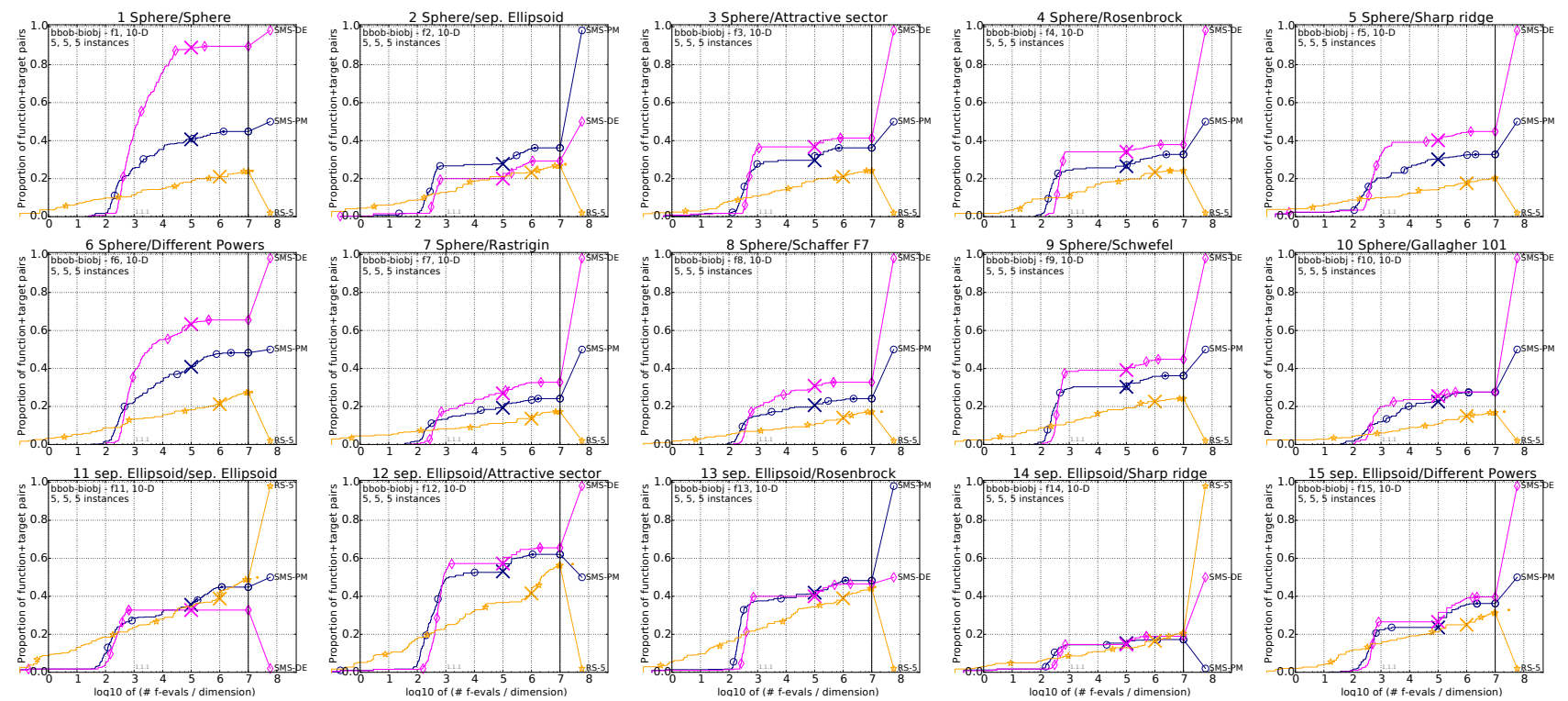

Figure 1: Bootstrapped empirical cumulative distribution of the number of objective function evaluations divided by dimension (FEvals/DIM) for 58 targets with target precision in $\left\{-10^{-4},-10^{-4.2}\right.$, $\left.-10^{-4.4},-10^{-4.6},-10^{-4.8},-10^{-5}, 0,10^{-5}, 10^{-4.9}, 10^{-4.8}, \ldots, 10^{-0.1}, 10^{0}\right\}$ for each single function $f_{1}$ to $f_{15}$ in 10-D.

functions, the pure random search is expected to take over for larger budgets which is related to the fact that on many functions, the performance of the SMS-EMOA algorithms flattens out or even does not improve anymore at some point. This indicates that the algorithm has converged. Whether all solutions have arrived close to the Pareto set cannot be decided from the data, while we believe that this is not the case most of the time. Then, restarts will likely be beneficial in terms of the performance measure underlying the $\mathrm{COCO}$ platform, namely the hypervolume indicator of the archive of all non-dominated solutions found so far [5].

\section{CONCLUSIONS}

We have compared numerically two variants of the SMSEMOA on the bbob-biobj test suite of the COCO platform. It turns out that the choice of the variation operators has a strong effect on the algorithm performance - with an advantage on almost all bbob-biobj test functions for the differential evolution variant over the polynomial mutation / simulated binary crossover version. This effect of the variation operator is expected to be present for other algorithms as well while its investigation is kept for future research.

\section{ACKNOWLEDGMENTS}

This work was supported by the grant ANR-12-MONU0009 (NumBBO) of the French National Research Agency.

\section{REFERENCES}

[1] A. Auger, D. Brockhoff, N. Hansen, D. Tušar, T. Tušar, and T. Wagner. Benchmarking the pure random search on the bi-objective BBOB-2016 testbed. In GECCO (Companion), 2016. DOI: 10.1145/2908961.2931704, to appear.

[2] A. Auger, D. Brockhoff, N. Hansen, D. Tušar, T. Tušar, and T. Wagner. The impact of search volume on the performance of RANDOMSEARCH on the bi-objective BBOB-2016 test suite. In GECCO (Companion), 2016. DOI: 10.1145/2908961.2931709, to appear.

[3] J. Bader and E. Zitzler. HypE: An Algorithm for Fast Hypervolume-Based Many-Objective Optimization. Evolutionary Computation, 19(1):45Ö-76, 2011.

[4] N. Beume, B. Naujoks, and M. Emmerich. SMS-EMOA: Multiobjective Selection Based on Dominated Hypervolume. European Journal of Operational Research, 181(3):1653-1669, 2007.

[5] D. Brockhoff, T. Tušar, D. Tušar, T. Wagner, N. Hansen, and A. Auger. Biobjective performance assessment with the COCO platform. ArXiv e-prints, arXiv:1605.01746, 2016.

[6] K. Deb and R. B. Agrawal. Simulated binary crossover for continuous search space. Complex systems, 9(2):115-148, 1995.

[7] K. Deb and M. Goyal. A combined genetic adaptive search (GeneAS) for engineering design. Computer Science and Informatics, 26:30-45, 1996.

[8] D. E. Goldberg. Genetic Algorithms in Search, Optimization, and Machine Learning. Addison-Wesley, Reading, Massachusetts, 1989.

[9] N. Hansen, A. Auger, D. Brockhoff, D. Tušar, and T. Tušar. COCO: Performance assessment. ArXiv e-prints, arXiv:1605.03560, 2016.

[10] N. Hansen, A. Auger, O. Mersmann, T. Tušar, and D. Brockhoff. COCO: A platform for comparing continuous optimizers in a black-box setting. ArXiv e-prints, arXiv:1603.08785, 2016.

[11] N. Hansen, T. Tušar, O. Mersmann, A. Auger, and D. Brockhoff. COCO: The experimental procedure. ArXiv e-prints, arXiv:1603.08776, 2016.

[12] C. Igel, N. Hansen, and S. Roth. Covariance Matrix 

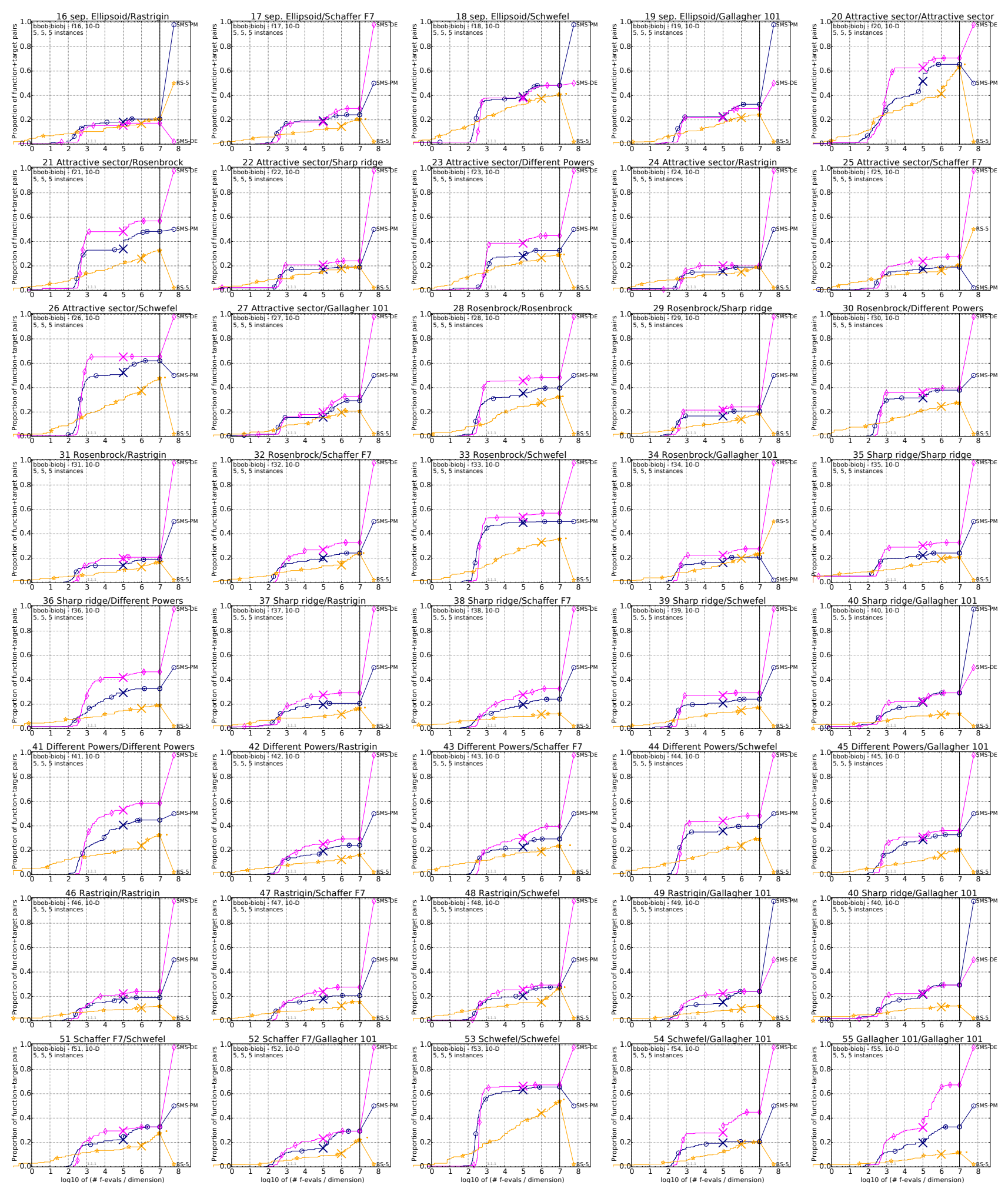

Figure 2: Bootstrapped empirical cumulative distribution of the number of objective function evaluations divided by dimension (FEvals/DIM) as in Fig. 1 but for functions $f_{16}$ to $f_{55}$ in 10-D. 
separable-separable

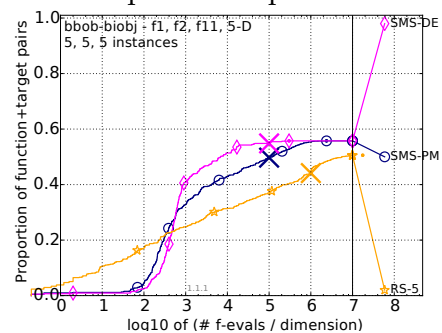

separable-weakstructure

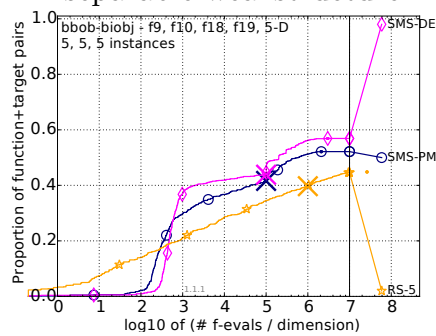

moderate-weakstructure

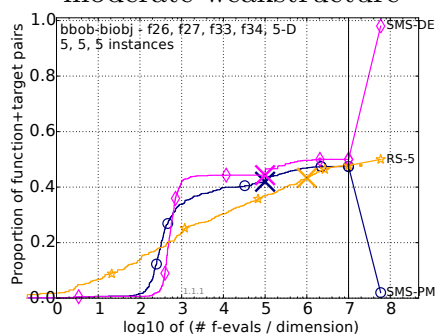

multimodal-multimodal

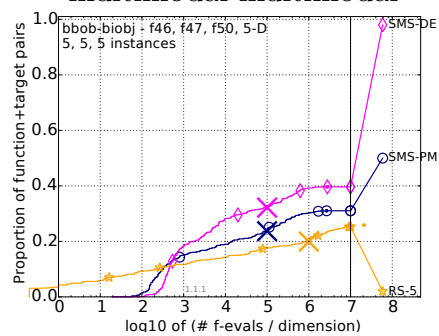

separable-moderate

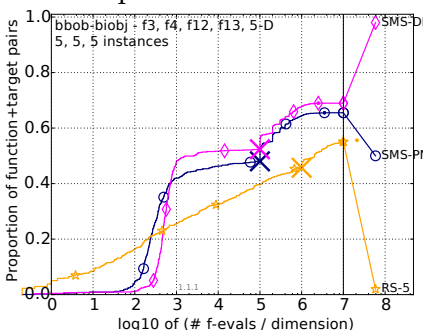

moderate-moderate

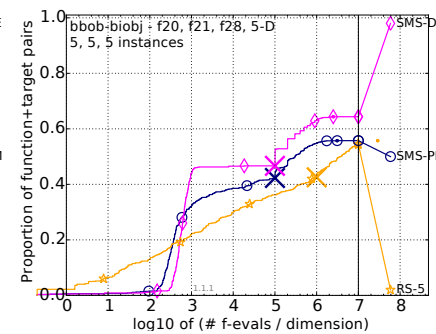

ill-cond.-ill-cond.

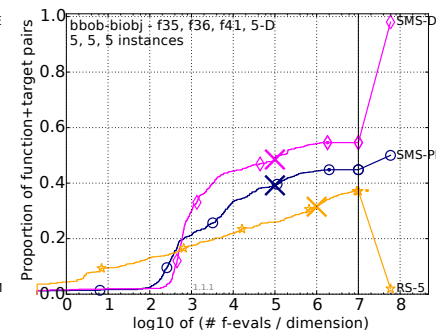

multimodal-weakstructure

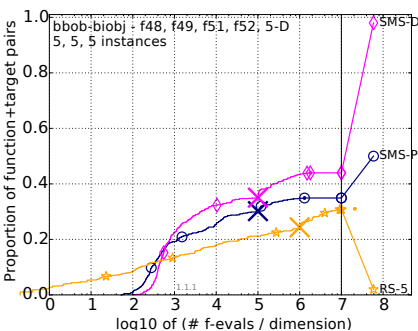

separable-ill-cond.

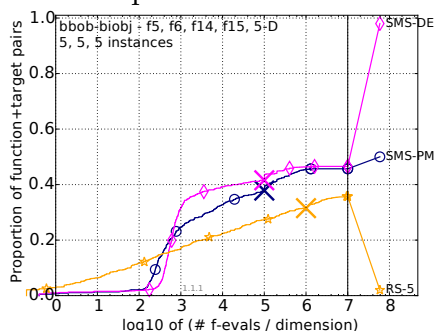

moderate-ill-cond.

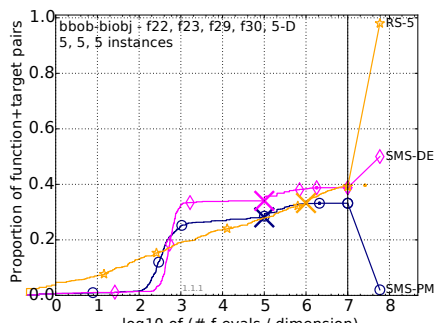

ill-cond.-multimodal

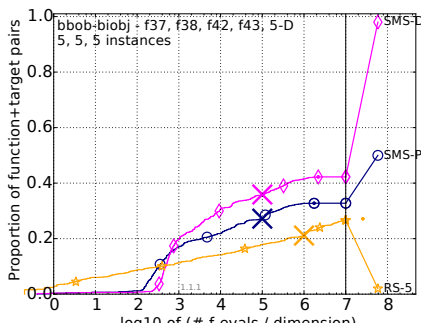

weakstructure-weakstructure

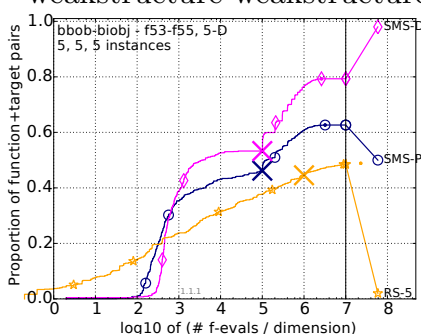

separable-multimodal

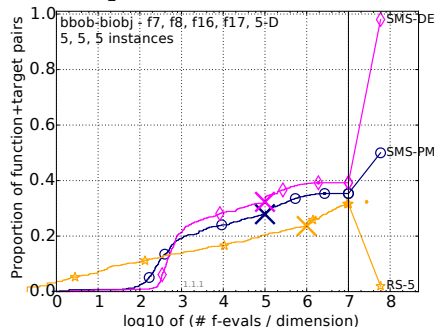

moderate-multimodal

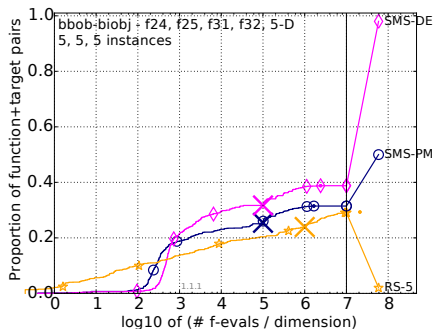

ill-cond.-weakstructure

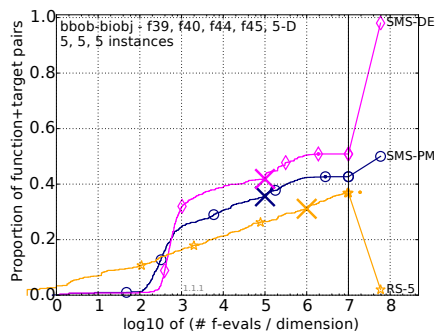

all 55 functions

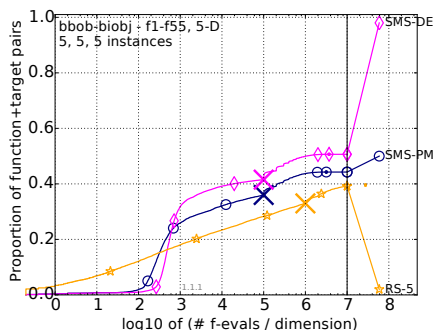

Figure 3: $\quad$ Bootstrapped empirical cumulative distribution of the number of objective function evaluations divided by dimension (FEvals/DIM) for 58 targets with target precision in $\left\{-10^{-4},-10^{-4.2}\right.$, $\left.-10^{-4.4},-10^{-4.6},-10^{-4.8},-10^{-5}, 0,10^{-5}, 10^{-4.9}, 10^{-4.8}, \ldots, 10^{-0.1}, 10^{0}\right\}$ for all functions and subgroups in 5 -D.

Adaptation for Multi-objective Optimization.

Evolutionary Computation, 15(1):1-28, 2007.

[13] K. Price. Differential evolution vs. the functions of the second ICEO. In Proceedings of the IEEE

International Congress on Evolutionary Computation, pages 153-157, 1997.

[14] R. Storn and K. Price. Differential evolution-a simple and efficient heuristic for global optimization over continuous spaces. Journal of global optimization, 11(4):341-359, 1997.
[15] T. Tušar, D. Brockhoff, N. Hansen, and A. Auger. COCO: The bi-objective black-box optimization benchmarking (bbob-biobj) test suite. ArXiv e-prints, arXiv:1604.00359, 2016. 
separable-separable

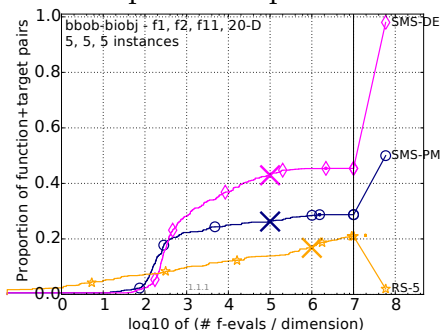

separable-weakstructure

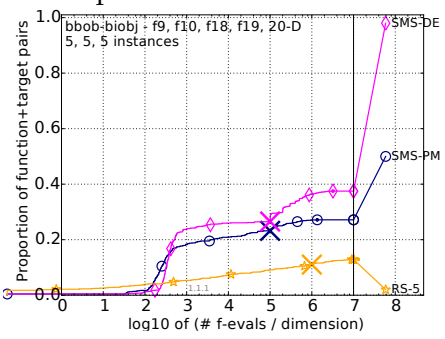

moderate-weakstructure

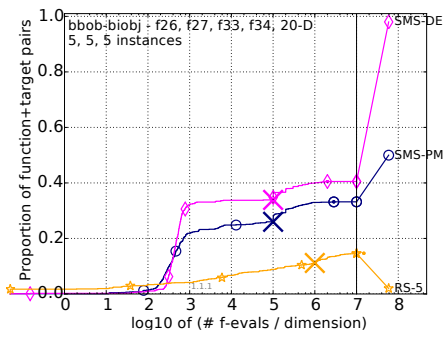

multimodal-multimodal

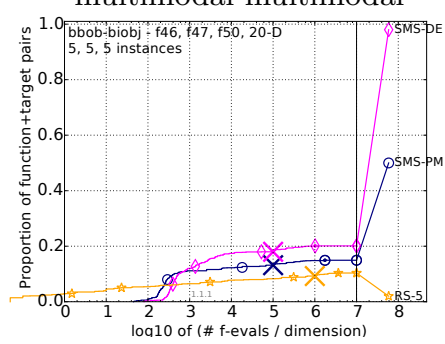

separable-moderate

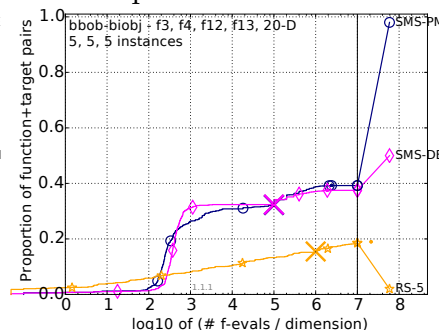

moderate-moderate

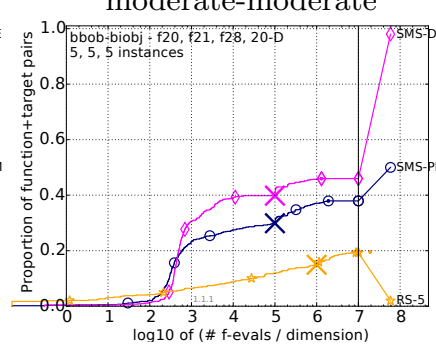

ill-cond.-ill-cond.

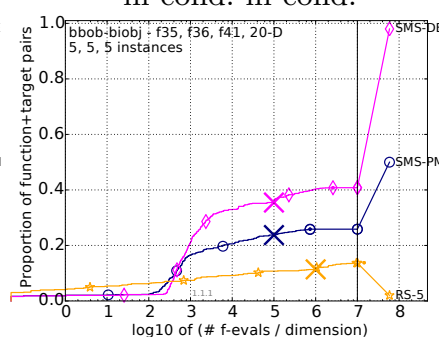

multimodal-weakstructure

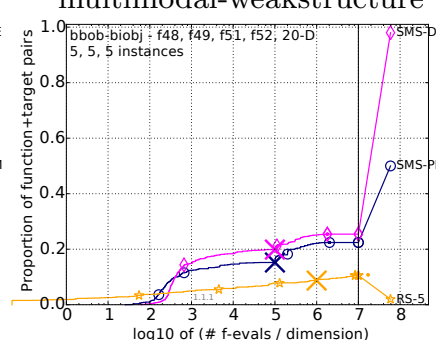

separable-ill-cond.

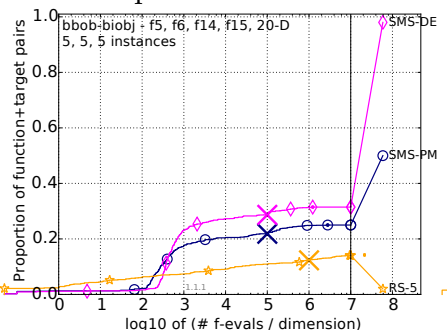

moderate-ill-cond.

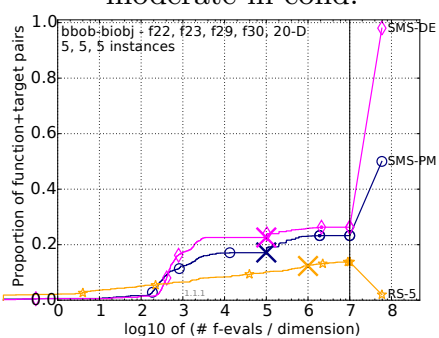

ill-cond.-multimodal

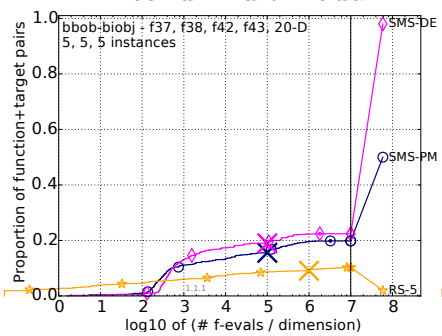

weakstructure-weakstructure

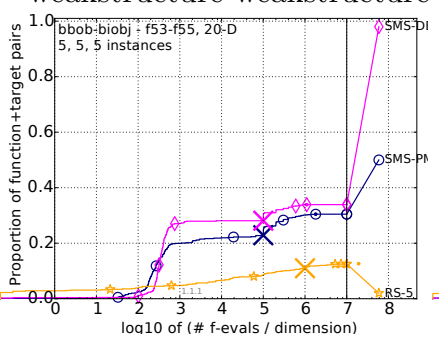

separable-multimodal

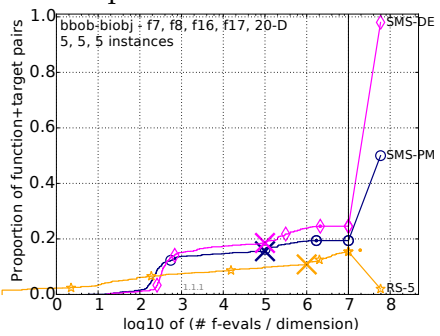

moderate-multimodal

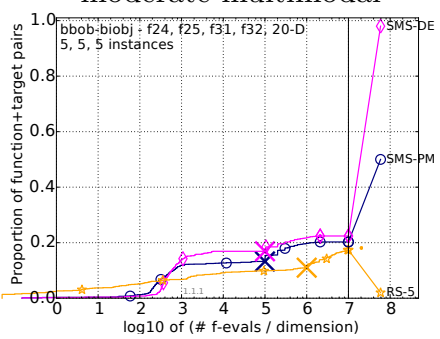

ill-cond.-weakstructure

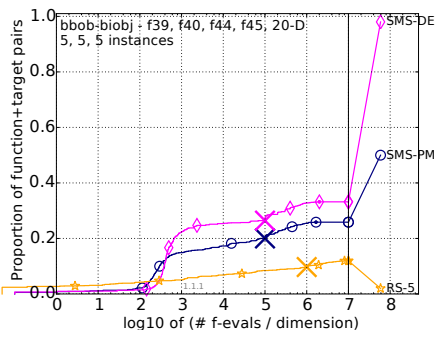

all 55 functions

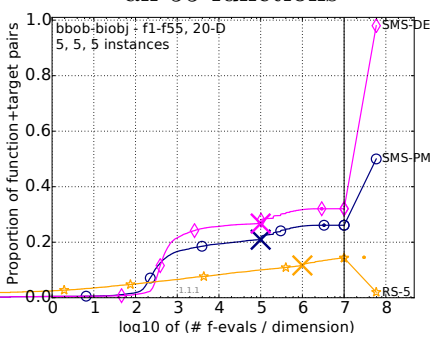

Figure 4: $\quad$ Bootstrapped empirical cumulative distribution of the number of objective function evaluations divided by dimension (FEvals/DIM) for 58 targets with target precision in $\left\{-10^{-4},-10^{-4.2}\right.$, $\left.-10^{-4.4},-10^{-4.6},-10^{-4.8},-10^{-5}, 0,10^{-5}, 10^{-4.9}, 10^{-4.8}, \ldots, 10^{-0.1}, 10^{0}\right\}$ for all functions and subgroups in 20-D. 


\begin{tabular}{|c|c|c|c|c|c|c|c|c|c|c|c|c|c|c|}
\hline$\Delta f$ & $1 \mathrm{e} 0$ & $1 \mathrm{e}-2$ & $1 e-5$ & $H x^{50}$ & $\Delta f$ & $1 \mathrm{e} 0$ & $1 \mathrm{e}-2$ & $1 e-5$ & $\mathbb{N}^{\mathrm{s}^{20}}$ & $\Delta f$ & $1 \mathrm{e} 0$ & $1 \mathrm{e}-2$ & $1 e-5$ & $\|^{s^{2}}$ \\
\hline f1 & & & & & f20 & & & & & f38 & & & & \\
\hline PM & $197(224)$ & $8480(2457)$ & $\infty 5$ es & $0 / 5$ & PM & $166(0.2)$ & $3.6 \mathrm{e} 5(3 \mathrm{e} 5)$ & $\infty 5 e 5$ & $0 / 5$ & PM & $239(185)$ & $\infty$ & $\infty 5 e 5$ & $0 / 5$ \\
\hline $\mathrm{DE}$ & $373(367)$ & $3173(229)$ & $\mathbf{9 . 3 e} 4(3176$ & $5 / 5$ & $\mathrm{DE}$ & $348(866)$ & $1.3 \mathrm{e} 5(5 \mathrm{e} 5)$ & $\infty 5 e 5$ & $0 / 5$ & $\mathrm{DE}$ & $602(346)$ & $\mathbf{5 . 5 e 5}(5 \mathrm{e} 5)$ & $\infty 5 \mathrm{e} 5$ & $0 / 5$ \\
\hline RS-5 & $\mathbf{1}(0)$ & $3.0 \mathrm{e} 6(1 \mathrm{e} 6)$ & $\infty 5$ e 6 & | $0 / 5$ & RS-5 & $\mathbf{1}(0)$ & $1.6 \mathrm{e} 6(5 \mathrm{e} 6)$ & $\infty 5 e 6$ & $0 / 5$ & RS-5 & $\mathbf{1}(0)$ & $\infty$ & $\infty 5 e 6$ & $0 / 5$ \\
\hline f2 & & & & & f21 & & & & & f39 & & & & \\
\hline$\overline{\mathrm{PM}}$ & $182(78)$ & $3.1 \mathrm{e} 4(4 \mathrm{e} 4)$ & $\infty 5 e 5$ & $0 / 5$ & $\overline{\mathrm{PM}}$ & $114(128)$ & $1.6 e 5(3 e 5)$ & $\infty 5 e 5$ & $0 / 5$ & $\overline{\mathrm{PM}}$ & $8.4(2)$ & $\infty$ & $\infty 5 e 5$ & $0 / 5$ \\
\hline $\mathrm{DE}$ & $190(305)$ & $1.3 \mathrm{e} 5(1 \mathrm{e} 5)$ & $\infty 5 e 5$ & $0 / 5$ & $\mathrm{DE}$ & $295(344)$ & $3.4 \mathrm{e} 5(6 \mathrm{e} 5)$ & $\infty 5 e 5$ & $0 / 5$ & $\mathrm{DE}$ & $8.4(2)$ & $\mathbf{2 . 0 e 6}(2 \mathrm{e} 6)$ & $\infty 5 e 5$ & $0 / 5$ \\
\hline RS-5 & $3.0(2)$ & $3.8 \mathrm{e} 6(4 \mathrm{e} 6)$ & $\infty 5 e 6$ & $0 / 5$ & RS-5 & $1(0)$ & $5.7 \mathrm{e} 6(3 \mathrm{e} 6)$ & $\infty 5 e 6$ & $0 / 5$ & RS-5 & $\mathbf{1}(0)$ & $\infty$ & $\infty 5 e 6$ & $0 / 5$ \\
\hline f3 & & & & & f22 & & & & & $\mathrm{f} 40$ & & & & i \\
\hline$\overline{\mathrm{PM}}$ & $64(133)$ & $7.8 \mathrm{e} 5(2 \mathrm{e} 6)$ & $\infty 5 e 5$ & $0 / 5$ & $\overline{\mathrm{PM}}$ & $1.6(0.5)$ & $\infty$ & $\infty 5 e 5$ & $0 / 5$ & $\overline{\mathrm{PM}}$ & $51(95)$ & $1.1 \mathrm{e} 6(1 \mathrm{e} 6)$ & $\infty 5 e 5$ & $0 / 5$ \\
\hline $\mathrm{DE}$ & $234(286)$ & $1.3 e 5(3 e 5)$ & $\infty 5 e 5$ & $0 / 5$ & $\mathrm{DE}$ & $1.6(0.5)$ & $\mathbf{2 . 0 e 6}(4 \mathrm{e} 6)$ & $\infty 5 e 5$ & $0 / 5$ & $\mathrm{DE}$ & $106(18)$ & $4.7 e 4(5289)$ & $\infty 5 \mathrm{e} 5$ & $0 / 5$ \\
\hline RS-5 & $\mathbf{1}(0)$ & $5.1 \mathrm{e} 6(5 \mathrm{e} 6)$ & $\infty 5 e 6$ & $0 / 5$ & RS-5 & $\mathbf{1}(0)$ & & $\infty 5 e 6$ & $0 / 5$ & RS-5 & $1(0)$ & $\infty$ & $\infty 5 e 6$ & $0 / 5$ \\
\hline f4 & & & & & f23 & & & & & f41 & & & & \\
\hline$\overline{\mathrm{PM}}$ & $398(154)$ & $8.0 \mathrm{e} 5(5 \mathrm{e} 5)$ & $\infty 5 e 5$ & $0 / 5$ & $\overline{\mathrm{PM}}$ & $15(18)$ & $7.6 \mathrm{e} 5(6 \mathrm{e} 5)$ & $\infty 5 e 5$ & $0 / 5$ & $\overline{\mathrm{PM}}$ & $478(168)$ & $2.2 \mathrm{e} 4(7055)$ & $\infty 5 e 5$ & $0 / 5$ \\
\hline $\mathrm{DE}$ & $765(268)$ & $3480(284)$ & $\infty 5 e 5$ & $0 / 5$ & $\mathrm{DE}$ & $16(10)$ & $1.3 \mathrm{e} 5(3 \mathrm{e} 5)$ & $\infty 5 e 5$ & $0 / 5$ & $\mathrm{DE}$ & $1041(578)$ & $5201(1499)$ & $\infty 5 e 5$ & $0 / 5$ \\
\hline RS-5 & $\mathbf{1}(0)$ & $1.4 \mathrm{e} 6(5 \mathrm{e} 5)$ & $\infty 5 e 6$ & $0 / 5$ & RS-5 & $1(0)$ & $3.8 \mathrm{e} 6(4 \mathrm{e} 6)$ & $\infty 5 e 6$ & $0 / 5$ & RS-5 & $3.8(4)$ & $3.6 \mathrm{e} 6(5 \mathrm{e} 6)$ & $\infty 5 e 6$ & $0 / 5$ \\
\hline f5 & & & & & f24 & & & & & f42 & & & & \\
\hline$\overline{\mathrm{PM}}$ & $5.6(2)$ & $5.0 \mathrm{e} 4(3 \mathrm{e} 4)$ & $\infty 5 e 5$ & $0 / 5$ & $\overline{\mathrm{PM}}$ & $100(18)$ & $\infty$ & $\infty 5 e 5$ & $0 / 5$ & $\overline{\mathrm{PM}}$ & $533(168)$ & $\infty$ & $\infty 5 e 5$ & $0 / 5$ \\
\hline $\mathrm{DE}$ & $5.2(4)$ & $1.3 \mathrm{e} 5(5 \mathrm{e} 5)$ & $\infty 5 e 5$ & $0 / 5$ & $\mathrm{DE}$ & $239(556)$ & $2.1 \mathrm{e} 6(2 \mathrm{e} 6)$ & $\infty 5 e 5$ & $0 / 5$ & $\mathrm{DE}$ & $1119(800)$ & $9.9 e 5(6 e 5)$ & $\infty 5 e 5$ & $0 / 5$ \\
\hline RS-5 & $1(0)$ & $\infty$ & $\infty 5 e 6$ & $0 / 5$ & RS-5 & $1.4(1)$ & $\infty$ & $\infty 5 e 6$ & $0 / 5$ & RS-5 & $3.0(2)$ & $\infty$ & $\infty 5 e 6$ & $0 / 5$ \\
\hline f6 & & & & & f25 & & & & & f 43 & & & & \\
\hline$\overline{\mathrm{PM}}$ & $304(254)$ & $1.5 \mathrm{e} 4(2 \mathrm{e} 4)$ & $\infty 5 e 5$ & $0 / 5$ & $\overline{\mathrm{PM}}$ & $151(193)$ & $2.2 \mathrm{e} 6(3 \mathrm{e} 6)$ & $\infty 5 e 5$ & $0 / 5$ & $\overline{\mathrm{PM}}$ & $628(186)$ & $8.7 \mathrm{e} 5(8 \mathrm{e} 5)$ & $\infty 5 e 5$ & $0 / 5$ \\
\hline $\mathrm{DE}$ & $581(421)$ & $\mathbf{3 8 4 6}(439)$ & $\infty 5 e 5$ & $0 / 5$ & $\mathrm{DE}$ & $311(382)$ & $4.1 \mathrm{e} 5(6 \mathrm{e} 5)$ & $\infty 5 e 5$ & $0 / 5$ & $\mathrm{DE}$ & $1310(227)$ & $1.3 e 5(1 \mathrm{e} 5)$ & $\infty 5 e 5$ & $0 / 5$ \\
\hline RS-5 & $\mathbf{1}(0)$ & $4.2 \mathrm{e} 6(4 \mathrm{e} 6)$ & $\infty 5 e 6$ & $0 / 5$ & RS-5 & $\mathbf{3 . 0 ( 5 )}$ & $\infty$ & $\infty 5 e 6$ & $0 / 5$ & RS-5 & 2.2(2) & $\infty$ & $\infty 5 e 6$ & $0 / 5$ \\
\hline f7 & & & & & f26 & & & & & f44 & & & & 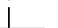 \\
\hline$\overline{\mathrm{PM}}$ & $200(244)$ & $2.4 \mathrm{e} 6(4 \mathrm{e} 6)$ & $\infty 5 e 5$ & $0 / 5$ & $\overline{\mathrm{PM}}$ & $153(232)$ & $1.4 \mathrm{e} 5(5 \mathrm{e} 5)$ & $\infty 5 e 5$ & $0 / 5$ & $\overline{\mathrm{PM}}$ & $521(347)$ & $8.9 \mathrm{e} 4(2 \mathrm{e} 5)$ & $\infty 5 e 5$ & $0 / 5$ \\
\hline $\mathrm{DE}$ & $288(137)$ & $2.0 e 5(4 \mathrm{e} 5)$ & $\infty 5 e 5$ & $0 / 5$ & $\mathrm{DE}$ & $560(697)$ & $\mathbf{3 3 6 5}(770)$ & $\infty 5 e 5$ & $0 / 5$ & $\mathrm{DE}$ & $935(497)$ & $\mathbf{3 3 6 5}(876)$ & $\infty 5 e 5$ & $0 / 5$ \\
\hline RS-5 & $\mathbf{1}(0)$ & $\infty$ & $\infty 5 e 6$ & $0 / 5$ & RS-5 & $\mathbf{3 . 6}(6)$ & $1.7 \mathrm{e} 6(3 \mathrm{e} 6)$ & $\infty 5 e 6$ & $0 / 5$ & RS-5 & $1(0)$ & $2.9 \mathrm{e} 6(3 \mathrm{e} 6)$ & $\infty 5 e 6$ & $0 / 5$ \\
\hline f8 & & & & & f 27 & & & & & f45 & & & & 1 \\
\hline$\overline{\mathrm{PM}}$ & $562(186)$ & $2.3 \mathrm{e} 6(2 \mathrm{e} 6)$ & $\infty 5 e 5$ & $0 / 5$ & $\mathrm{PM}$ & $30(40)$ & $2.0 \mathrm{e} 6(2 \mathrm{e} 6)$ & $\infty 5 e 5$ & $0 / 5$ & $\overline{\mathrm{PM}}$ & $507(329)$ & $\mathbf{6 . 8 e} 4(6 \mathrm{e} 4)$ & $\infty 5 e 5$ & $0 / 5$ \\
\hline $\mathrm{DE}$ & $1159(119)$ & $1.7 \mathrm{e} 5(2 \mathrm{e} 5)$ & $\infty 5 e 5$ & $0 / 5$ & $\mathrm{DE}$ & $30(38)$ & $3.4 \mathrm{e} 5(6 \mathrm{e} 5)$ & $\infty 5 e 5$ & $0 / 5$ & $\mathrm{DE}$ & $871(597)$ & $1.4 \mathrm{e} 5(1 \mathrm{e} 5)$ & $\infty 5 e 5$ & $0 / 5$ \\
\hline RS-5 & $\mathbf{3 . 8}(0)$ & $\infty$ & $\infty 5 e 6$ & $0 / 5$ & RS-5 & $2.0(2)$ & $1.7 \mathrm{e} 6(2 \mathrm{e} 6)$ & $\infty 5 \mathrm{e} 6$ & $0 / 5$ & RS-5 & $3.8(6)$ & $2.4 \mathrm{e} 7(2 \mathrm{e} 7)$ & $\infty 5 e 6$ & $0 / 5$ \\
\hline f9 & & & & & f28 & & & & & f46 & & & & i \\
\hline$\overline{\mathrm{PM}}$ & $357(177)$ & $6.3 \mathrm{e} 4(1 \mathrm{e} 5)$ & $\infty 5 e 5$ & $0 / 5$ & $\overline{\mathrm{PM}}$ & $275(59)$ & $2.9 \mathrm{e} 4(3 \mathrm{e} 4)$ & $\infty 5 e 5$ & $0 / 5$ & $\overline{\mathrm{PM}}$ & $362(182)$ & $\infty$ & $\infty 5 e 5$ & $0 / 5$ \\
\hline $\mathrm{DE}$ & $705(550)$ & $\mathbf{3 1 2 5}(314)$ & $\infty 5 e 5$ & $0 / 5$ & $\mathrm{DE}$ & $531(198)$ & $\mathbf{3 1 1 8}(464)$ & $\infty 5 e 5$ & $0 / 5$ & $\mathrm{DE}$ & $716(588)$ & $\infty$ & $\infty 5 e 5$ & $0 / 5$ \\
\hline RS-5 & $2.4(4)$ & $2.4 \mathrm{e} 6(4 \mathrm{e} 6)$ & $\infty 5 e 6$ & $0 / 5$ & RS-5 & $1(0)$ & $7.4 \mathrm{e} 5(2 \mathrm{e} 6)$ & $\infty 5 e 6$ & $0 / 5$ & RS-5 & 10 & $\infty$ & $\infty 5 e 6$ & $0 / 5$ \\
\hline f10 & & & & & f29 & & & & & f47 & & & & 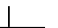 \\
\hline$\overline{\mathrm{PM}}$ & $339(107)$ & $1.8 \mathrm{e} 5(3 \mathrm{e} 5)$ & $\infty 5 e 5$ & $0 / 5$ & $\overline{\mathrm{PM}}$ & $233(170)$ & $\infty$ & $\infty 5 e 5$ & $0 / 5$ & $\overline{\mathrm{PM}}$ & $460(98)$ & $\infty$ & $\infty 5 e 5$ & $0 / 5$ \\
\hline $\mathrm{DE}$ & $660(127)$ & $1.5 \mathrm{es}(2 \mathrm{e} 5)$ & $\infty 5 e 5$ & $0 / 5$ & $\mathrm{DE}$ & $402(433)$ & $\infty$ & $\infty 5 e 5$ & $0 / 5$ & $\mathrm{DE}$ & $908(246)$ & $2.1 \mathrm{e}(2 \mathrm{e} 6)$ & $\infty 5$ e 5 & $0 / 5$ \\
\hline RS-5 & $\mathbf{1}(0)$ & $2.3 \mathrm{e} 7(2 \mathrm{e} 7)$ & $\infty 5$ e 6 & $0 / 5$ & RS-5 & $1(0)$ & $\infty$ & $\infty 5 e 6$ & $0 / 5$ & RS-5 & $1(0)$ & $\infty$ & $\infty 5 e 6$ & $0 / 5$ \\
\hline f11 & & & & & f3o & & & & & f48 & & & & \\
\hline$\overline{\mathrm{PM}}$ & $1.2(0.5)$ & $2.4 \mathrm{e} 4(6 \mathrm{e} 4)$ & $\infty 5 e 5$ & $0 / 5$ & $\overline{\mathrm{PM}}$ & $569(176)$ & $7.9 \mathrm{e} 5(9 \mathrm{e} 5)$ & $\infty 5 e 5$ & $0 / 5$ & $\overline{\mathrm{PM}}$ & $703(292)$ & $\mathbf{1 . 0 e 6}(1 \mathrm{e} 6)$ & $\infty 5 e 5$ & $0 / 5$ \\
\hline $\mathrm{DE}$ & $1.2(0)$ & $\mathbf{2 2 2 5}(450)$ & $\infty 5 e 5$ & $0 / 5$ & $\mathrm{DE}$ & $1014(264)$ & $1.3 \mathrm{e} 5(6 \mathrm{e} 5)$ & $\infty 5 e 5$ & $0 / 5$ & $\mathrm{DE}$ & $1232(290)$ & $2.0 \mathrm{e} 6(3 \mathrm{e} 6)$ & $\infty 5 e 5$ & $0 / 5$ \\
\hline RS-5 & $1(0)$ & $4.5 \mathrm{e} 5(1 \mathrm{e} 6)$ & $\infty 5 e 6$ & $0 / 5$ & RS-5 & $\mathbf{2 . 0}(2)$ & $3.8 \mathrm{e} 6(4 \mathrm{e} 6)$ & $\infty 5 e 6$ & $0 / 5$ & RS-5 & $\mathbf{5 . 6 ( 4 )}$ & $\infty$ & $\infty 5 e 6$ & $0 / 5$ \\
\hline f12 & & & & & f31 & & & & & f 49 & & & & L \\
\hline$\overline{\mathrm{PM}}$ & $65(82)$ & $1.3 e 5(1 \mathrm{e} 5)$ & $\infty 5 e 5$ & $0 / 5$ & $\overline{\mathrm{PM}}$ & $450(150)$ & $\infty$ & $\infty 5 e 5$ & $0 / 5$ & $\overline{\mathrm{PM}}$ & $442(181)$ & $\infty$ & $\infty 5 e 5$ & $0 / 5$ \\
\hline $\mathrm{DE}$ & $145(260)$ & $1.3 \mathrm{e} 5(5 \mathrm{e} 5)$ & $2.0 \mathrm{e} 6(2 \mathrm{e} 6)$ & $1 / 5$ & $\mathrm{DE}$ & $767(588)$ & $8.0 e 5(1 \mathrm{e} 6)$ & $\infty 5 e 5$ & $0 / 5$ & $\mathrm{DE}$ & $787(229)$ & $8.0 e 5(1 \mathrm{e} 6)$ & $\infty 5 e 5$ & $0 / 5$ \\
\hline RS-5 & $\mathbf{1}(0)$ & $2.1 \mathrm{e} 6(2 \mathrm{e} 6)$ & $\infty 5 e 6$ & $0 / 5$ & RS-5 & $\mathbf{1}(0)$ & $\infty$ & $\infty 5 e 6$ & $0 / 5$ & RS-5 & $2.0(2)$ & $\infty$ & $\infty 5 e 6$ & $0 / 5$ \\
\hline f13 & & & & & f32 & & & & & f5o & & & & 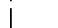 \\
\hline$\overline{\mathrm{PM}}$ & $220(272)$ & 1583(390) & $\infty 5 e 5$ & $0 / 5$ & $\overline{\mathrm{PM}}$ & \begin{tabular}{|l}
$587(101)$ \\
\end{tabular} & $\infty$ & $\infty 5 e 5$ & $0 / 5$ & $\overline{\mathrm{PM}}$ & $541(122)$ & $2.5 \mathrm{e} 6(4 \mathrm{e} 6)$ & $\infty 5 e 5$ & $0 / 5$ \\
\hline $\mathrm{DE}$ & $573(582)$ & $2954(386)$ & $\infty 5 e 5$ & $0 / 5$ & $\mathrm{DE}$ & $1130(270)$ & $\mathbf{8 . 2 e 5}(8 \mathrm{e} 5)$ & $\infty 5 e 5$ & $0 / 5$ & $\mathrm{DE}$ & $1084(332)$ & $1.5 e 5(1 \mathrm{e} 5)$ & $\infty 5 e 5$ & $0 / 5$ \\
\hline $\mathrm{RS}-5$ & $\mathbf{2 . 8}(4)$ & $1.3 \mathrm{e} 5(2 \mathrm{e} 5)$ & $\infty 5 e 6$ & $0 / 5$ & $\mathrm{RS}-5$ & $3.8(2)$ & $\infty$ & $\infty 5 e 6$ & $0 / 5$ & RS-5 & $1(0)$ & $\infty$ & $\infty 5 e 6$ & $0 / 5$ \\
\hline f14 & & & & & f33 & & & & & f51 & & & & \\
\hline$\overline{\mathrm{PM}}$ & $2.0(0)$ & $\infty$ & $\infty 5 e 5$ & $0 / 5$ & $\overline{\mathrm{PM}}$ & $388(138)$ & $1949(856)$ & $\infty 5 e 5$ & $0 / 5$ & $\overline{\mathrm{PM}}$ & $558(130)$ & $8.2 \mathrm{e} 5(1 \mathrm{e} 6)$ & $\infty 5 e 5$ & $0 / 5$ \\
\hline $\mathrm{DE}$ & $2.0(0)$ & $\infty$ & $\infty 5 \mathrm{e} 5$ & $0 / 5$ & $\mathrm{DE}$ & $1010(741)$ & $2461(78)$ & $\infty 5 e 5$ & $0 / 5$ & $\mathrm{DE}$ & $1032(428)$ & $3.5 \mathrm{e} 4(3 \mathrm{e} 4)$ & $\infty 5 e 5$ & $0 / 5$ \\
\hline RS-5 & $1(0)$ & $\infty$ & $\infty 5$ e6 & $0 / 5$ & $\mathrm{RS}-5$ & $4.6(0)$ & $2.4 \mathrm{e} 4(2 \mathrm{e} 4)$ & $\infty 5$ e 6 & $0 / 5$ & RS-5 & $1.2(0.5)$ & $\infty$ & $\infty 5 e 6$ & $0 / 5$ \\
\hline f15 & & & & & f34 & & & & & f52 & & & & $\downarrow$ \\
\hline $\mathrm{PM}$ & $280(220)$ & $7.6 \mathrm{e} 5(4 \mathrm{e} 5)$ & $\infty 5 e 5$ & $0 / 5$ & $\overline{\mathrm{PM}}$ & $393(84)$ & $\infty$ & $\infty 5 e 5$ & $0 / 5$ & $\overline{\mathrm{PM}}$ & $507(270)$ & $\infty$ & $\infty 5 e 5$ & $0 / 5$ \\
\hline $\mathrm{DE}$ & $634(918)$ & $7.6 e 5(2 \mathrm{e} 6)$ & $\infty 5 e 5$ & $0 / 5$ & $\mathrm{DE}$ & $880(138)$ & $\mathbf{7 . 5 e 5}(8 \mathrm{e} 5)$ & $\infty 5 e 5$ & $0 / 5$ & $\mathrm{DE}$ & $999(138)$ & $2.0 \mathrm{e} 6(2 \mathrm{e} 6)$ & $\infty 5 e 5$ & $0 / 5$ \\
\hline RS-5 & $\mathbf{6 . 4 ( 6 )}$ & $8.4 \mathrm{e} 6(9 \mathrm{e} 6)$ & $\infty 5 e 6$ & $0 / 5$ & RS-5 & $1.6(0.5)$ & $1.6 \mathrm{e} 6(3 \mathrm{e} 6)$ & $\infty 5 e 6$ & $0 / 5$ & RS-5 & $1.4(0.5)$ & $2.1 \mathrm{e} 7(3 \mathrm{e} 7)$ & $\infty 5 e 6$ & $0 / 5$ \\
\hline f16 & & & & & f35 & & & & & f53 & & & & i \\
\hline$\overline{\mathrm{PM}}$ & $65(127)$ & $\infty$ & $\infty 5 e 5$ & $0 / 5$ & $\overline{\mathrm{PM}}$ & $1(0)$ & $\infty$ & $\infty 5 e 5$ & $0 / 5$ & $\overline{\mathrm{PM}}$ & $321(294)$ & $\mathbf{1 6 1 3}(460)$ & $\infty 5 e 5$ & $0 / 5$ \\
\hline $\mathrm{DE}$ & $42(72)$ & $\infty$ & $\infty 5 e 5$ & $0 / 5$ & $\mathrm{DE}$ & $1(0)$ & $\infty$ & $\infty 5 e 5$ & $0 / 5$ & $\mathrm{DE}$ & $650(552)$ & $2302(194)$ & $2.0 e 6(2 \mathrm{e} 6)$ & $1 / 5$ \\
\hline RS-5 & $2.0(2)$ & $2.2 \mathrm{e} 7(2 \mathrm{e} 7)$ & $\infty 5 e 6$ & $0 / 5$ & $\mathrm{RS}-5$ & $1(0)$ & $\mathbf{2 . 3 e 7}(2 \mathrm{e} 7)$ & $\infty 5 e 6$ & $0 / 5$ & RS-5 & $20(24)$ & $1.8 \mathrm{e} 4(2 \mathrm{e} 4)$ & $\infty 5 e 6$ & $0 / 5$ \\
\hline f17 & & & & & f36 & & & & & f54 & & & & \\
\hline$\overline{\mathrm{PM}}$ & $195(371)$ & $2.2 \mathrm{e} 6(3 \mathrm{e} 6)$ & $\infty 5 e 5$ & $0 / 5$ & $\overline{\mathrm{PM}}$ & $83(166)$ & $4.7 \mathrm{e} 5(5 \mathrm{e} 5)$ & $\infty 5 e 5$ & $0 / 5$ & $\overline{\mathrm{PM}}$ & \begin{tabular}{|c|}
$348(180)$ \\
\end{tabular} & $8.5 \mathrm{e} 5(8 \mathrm{e} 5)$ & $\infty 5 e 5$ & $0 / 5$ \\
\hline $\mathrm{DE}$ & $584(718)$ & $2.0 \mathrm{e}(2 \mathrm{e} 6)$ & $\infty 5 e 5$ & $0 / 5$ & $\mathrm{DE}$ & $153(185)$ & $2.6 e 5(5 e 5)$ & $\infty 5 e 5$ & $0 / 5$ & $\mathrm{DE}$ & $1077(124)$ & $\mathbf{3 . 4 e 5}(6 \mathrm{e} 5)$ & $\infty 5 e 5$ & $0 / 5$ \\
\hline RS-5 & $1702(2)$ & $\infty$ & $\infty 5 e 6$ & $0 / 5$ & RS-5 & $\mathbf{1}(0)$ & $\infty$ & $\infty 5 e 6$ & $0 / 5$ & RS-5 & $1(0)$ & $9.9 \mathrm{e} 5(5 \mathrm{e} 5)$ & $\infty 5 e 6$ & $0 / 5$ \\
\hline f18 & & & & & f37 & & & & & f55 & & & & \\
\hline $\mathrm{PM}$ & $4.8(4)$ & $1.1 \mathrm{e} 4(2 \mathrm{e} 4)$ & $\infty 5 e 5$ & $0 / 5$ & $\overline{\mathrm{PM}}$ & $7.4(10)$ & $\infty$ & $\infty 5 e 5$ & $0 / 5$ & $\overline{\mathrm{PM}}$ & $447(183)$ & $2.0 \mathrm{e} 6(2 \mathrm{e} 6)$ & $\infty 5 e 5$ & $0 / 5$ \\
\hline $\mathrm{DE}$ & $3.2(4)$ & $\mathbf{2 6 8 8}(196)$ & $\infty 5 e 5$ & $0 / 5$ & $\mathrm{DE}$ & $7.4(2)$ & $9.3 \mathrm{e} 5(1 \mathrm{e} 6)$ & $\infty 5 e 5$ & $0 / 5$ & $\mathrm{DE}$ & $638(272)$ & $\mathbf{7 . 8 e 5}(1 \mathrm{e} 6)$ & $2.1 \mathrm{e} 6(3 \mathrm{e} 6)$ & $1 / 5$ \\
\hline RS-5 & $1(0)$ & $4.2 \mathrm{e} 5(5 \mathrm{e} 5)$ & $\infty 5 e 6$ & $0 / 5$ & RS-5 & $1(0)$ & $\infty$ & $\infty 5 e 6$ & $0 / 5$ & RS-5 & $1(0)$ & $2.2 \mathrm{e} 7(2 \mathrm{e} 7)$ & $\infty 5 e 6$ & $0 / 5$ \\
\hline f19 & & & & & & & & & & & & & & \\
\hline$\overline{\mathrm{PM}}$ & $147(115)$ & $2.0 \mathrm{e} 6(2 \mathrm{e} 6)$ & $\infty 5 e 5$ & $0 / 5$ & & & & & & & & & & \\
\hline $\mathrm{DE}$ & $383(359)$ & $2.0 \mathrm{e} 6(3 \mathrm{e} 6)$ & $\infty 5 e 5$ & $0 / 5$ & & & & & & & & & & \\
\hline RS-5 & $1.8(1)$ & $5.5 e 5(2 \mathrm{e} 5)$ & $\infty 5 e 6$ & $0 / 5$ & & & & & & & & & & \\
\hline
\end{tabular}

Table 2: Average runtime (aRT) to reach given targets, measured in number of function evaluations, in dimension 5. For each function, the aRT and, in braces as dispersion measure, the half difference between 10 and 90\%-tile of (bootstrapped) runtimes is shown for the different target $\Delta I$-values as shown in the top row. \#succ is the number of trials that reached the last target $I^{\text {ref }}+10^{-5}$. The median number of conducted function evaluations is additionally given in italics, if the target in the last column was never reached. Entries, succeeded by a star, are statistically significantly better (according to the rank-sum test) when compared to all other algorithms of the table, with $p=0.05$ or $p=10^{-k}$ when the number $k$ following the star is larger than 1, with Bonferroni correction of 110. Best results are printed in bold. 


\begin{tabular}{|c|c|c|c|c|c|c|c|c|c|c|c|c|c|c|}
\hline$\Delta f$ & $1 \mathrm{e} 0$ & $1 \mathrm{e}-2$ & $1 e-5$ & $H^{55^{20}}$ & $\Delta f$ & $1 \mathrm{e} 0$ & $1 \mathrm{e}-2$ & $1 e-5$ & $\mathbb{H}^{5^{20}}$ & $\Delta f$ & $1 \mathrm{e} 0$ & $1 \mathrm{e}-2$ & $1 e-5$ & $\mathbb{A}^{s^{20}}$ \\
\hline f1 & & & & & $\mathrm{f20}$ & & & & & $\begin{array}{l}\mathrm{f38} \\
\end{array}$ & & & & \\
\hline PM & $525(86)$ & $\infty$ & $\infty 2 e 6$ & $0 / 5$ & PM & $77(167)$ & $8.0 \mathrm{e} 6(4 \mathrm{e} 6)$ & $\infty 2 e 6$ & $0 / 5$ & PM & $1202(414)$ & $\infty$ & $\infty 2 e 6$ & $0 / 5$ \\
\hline $\mathrm{DE}$ & $1201(244)$ & $1.4 \mathrm{e} 4(1787)$ & 9.6e6 $(9 e 6)$ & $1 / 5$ & $\mathrm{DE}$ & $110(249)$ & $5.6 \mathrm{e} 5(2 \mathrm{e} 4)$ & $\infty 2 e 6$ & $0 / 5$ & $\mathrm{DE}$ & $2402(59)$ & $\infty$ & $\infty 2 e 6$ & $0 / 5$ \\
\hline RS-5 & $1(0)$ & $\infty$ & $\infty 2 e 7$ & $0 / 5$ & RS-5 & $\mathbf{1}(0)$ & $\infty$ & $\infty 2 e 7$ & $0 / 5$ & RS-5 & $1(0)$ & $\infty$ & $\infty 2 e 7$ & $10 / 5$ \\
\hline f2 & & & & & f21 & & & & & f39 & & & & \\
\hline$\overline{\mathrm{PM}}$ & 609(308) & $\infty$ & $\infty 2 e 6$ & $0 / 5$ & $\overline{\mathrm{PM}}$ & $538(351)$ & $\infty$ & $\infty 2 e 6$ & $0 / 5$ & $\overline{\mathrm{PM}}$ & $62(70)$ & $\infty$ & $\infty 2 e 6$ & $0 / 5$ \\
\hline DE & $1127(319)$ & $\infty$ & $\infty 2 e 6$ & $0 / 5$ & $\mathrm{DE}$ & $1227(704)$ & $5.1 \mathrm{e} 5(2 \mathrm{e} 6)$ & $\infty 2 e 6$ & $0 / 5$ & $\mathrm{DE}$ & $84(187)$ & $\infty$ & $\infty 2 e 6$ & $0 / 5$ \\
\hline RS-5 & $1(0)$ & $\infty$ & $\infty 2 e^{7}$ & $0 / 5$ & RS-5 & $\mathbf{1}(0)$ & $\infty$ & $\infty 2 e 7$ & $0 / 5$ & RS-5 & $1(0)$ & $\infty$ & $\infty 2 e 7$ & $0 / 5$ \\
\hline f3 & & & & $\downarrow$ & f22 & & & & & f40 & & & & $\downarrow$ \\
\hline$\overline{\mathrm{PM}}$ & $155(75)$ & $\infty$ & $\infty 2 e 6$ & $0 / 5$ & $\overline{\mathrm{PM}}$ & $1.6(1)$ & $\infty$ & $\infty 2 e 6$ & $0 / 5$ & $\overline{\mathrm{PM}}$ & $9.0(18)$ & $\infty$ & $\infty 2 e 6$ & $0 / 5$ \\
\hline $\mathrm{DE}$ & $284(488)$ & $\infty$ & $\infty 2 e 6$ & $0 / 5$ & $\mathrm{DE}$ & $1.6(1)$ & $\infty$ & $\infty 2 e 6$ & $0 / 5$ & $\mathrm{DE}$ & $9.0(18)$ & $\infty$ & $\infty 2 e 6$ & $0 / 5$ \\
\hline RS-5 & $\mathbf{1}(0)$ & $\infty$ & $\infty 2 e 7$ & $0 / 5$ & RS-5 & $\mathbf{1}(0)$ & $\infty$ & $\infty 2 e 7$ & $0 / 5$ & $\mathrm{RS}-5$ & $\mathbf{1}(0)$ & $\infty$ & $\infty 2 e^{7}$ & $0 / 5$ \\
\hline f4 & & & & i & f23 & & & & & f41 & & & & \\
\hline$\overline{\mathrm{PM}}$ & $1567(406)$ & $\infty$ & $\infty 2 e 6$ & $0 / 5$ & $\overline{\mathrm{PM}}$ & $359(152)$ & $\infty$ & $\infty 2 e 6$ & $0 / 5$ & $\mathrm{PM}$ & $4012(1168)$ & $\infty$ & $\infty 2 e 6$ & $0 / 5$ \\
\hline $\mathrm{DE}$ & $3144(406)$ & $\infty$ & $\infty 2 e 6$ & $0 / 5$ & $\mathrm{DE}$ & $708(498)$ & $\infty$ & $\infty 2 e 6$ & $0 / 5$ & $\mathrm{DE}$ & $6485(1111)$ & 6.0e4(5939) & )$\infty 2 e 6$ & $0 / 5$ \\
\hline RS-5 & $\mathbf{1}(0)$ & $\infty$ & $\infty 2 e 7$ & $0 / 5$ & RS-5 & $1.2(0.5)$ & $\infty$ & $\infty 2 e 7$ & $0 / 5$ & RS-5 & $1.2(0.5)$ & $\infty$ & $\infty 2 e 7$ & $0 / 5$ \\
\hline f5 & & & & & f24 & & & & & f42 & & & & \\
\hline$\overline{\mathrm{PM}}$ & $1.4(0.5)$ & $\infty$ & $\infty 2 e 6$ & $0 / 5$ & $\overline{\mathrm{PM}}$ & $107(9)$ & $\infty$ & $\infty 2 e 6$ & $0 / 5$ & $\overline{\mathrm{PM}}$ & $2862(1077)$ & $\infty$ & $\infty 2 e 6$ & $0 / 5$ \\
\hline $\mathrm{DE}$ & $1.4(0.5)$ & $1.9 \mathrm{e} 5(1 \mathrm{e} 5)$ & $\infty 2 e 6$ & $0 / 5$ & $\mathrm{DE}$ & $180(216)$ & $\infty$ & $\infty 2 e 6$ & $0 / 5$ & $\mathrm{DE}$ & $4875(988)$ & $\infty$ & $\infty 2 e 6$ & $0 / 5$ \\
\hline RS-5 & $1(0)$ & $\infty$ & $\infty 2 e 7$ & $0 / 5$ & RS-5 & $1(0)$ & $\infty$ & $\infty 2 e 7$ & $0 / 5$ & RS-5 & $1(0)$ & $\infty$ & $\infty 2 e 7$ & $0 / 5$ \\
\hline f6 & & & & j & f25 & & & & & $\mathrm{f} 43$ & & & & j \\
\hline$\overline{\mathrm{PM}}$ & $1216(374)$ & $\infty$ & $\infty 2 e 6$ & $0 / 5$ & $\overline{\mathrm{PM}}$ & $801(216)$ & $\infty$ & $\infty 2 e 6$ & $0 / 5$ & $\overline{\mathrm{PM}}$ & $3094(88)$ & $\infty$ & $\infty 2 e 6$ & $0 / 5$ \\
\hline $\mathrm{DE}$ & $2694(930)$ & $\mathbf{2 . 4 e} 4(6796)$ & )$\infty 2 e 6$ & $0 / 5$ & $\mathrm{DE}$ & $1579(934)$ & $\infty$ & $\infty 2 e 6$ & $0 / 5$ & $\mathrm{DE}$ & $5232(290)$ & $\infty$ & $\infty 2 e 6$ & $0 / 5$ \\
\hline RS-5 & 10 & $\infty$ & $\infty 2 e 7$ & $0 / 5$ & RS-5 & $\mathbf{1 . 2}(0.5)$ & $\infty$ & $\infty 2 e 7$ & $0 / 5$ & RS-5 & $1(0)$ & $\infty$ & $\infty 2 e 7$ & $0 / 5$ \\
\hline f7 & & & & i & f26 & & & & a & f44 & & & & - \\
\hline$\overline{\mathrm{PM}}$ & \begin{tabular}{|l|}
$548(129)$ \\
\end{tabular} & $\infty$ & $\infty 2 e 6$ & $0 / 5$ & $\overline{\mathrm{PM}}$ & $1030(951)$ & $1.4 \mathrm{e} 6(2 \mathrm{e} 6)$ & $\infty 2 e 6$ & $0 / 5$ & $\overline{\mathrm{PM}}$ & $2313(541)$ & $\infty$ & $\infty 2 e 6$ & $0 / 5$ \\
\hline DE & $1448(858)$ & $\infty$ & $\infty 2 e 6$ & $0 / 5$ & $\mathrm{DE}$ & $2228(2683)$ & $1.2 \mathrm{e} 4(2015)$ & )$\infty 2 e 6$ & $0 / 5$ & $\mathrm{DE}$ & $4603(1290)$ & $8.0 e 6(6 e 6)$ & $\infty 2 e 6$ & $0 / 5$ \\
\hline RS-5 & 1 $1(0)$ & $\infty$ & $\infty 2 e 7$ & $0 / 5$ & RS-5 & $\mathbf{1}(0)$ & $\infty$ & $\infty 2 e 7$ & $0 / 5$ & RS-5 & $2.0(2)$ & $\infty$ & $\infty 2 e 7$ & $10 / 5$ \\
\hline f8 & & & & $\mid$ & f27 & & & & & f45 & & & & 1 \\
\hline$\overline{\mathrm{PM}}$ & $1852(264)$ & $\infty$ & $\infty 2 e 6$ & $0 / 5$ & $\mathrm{PM}$ & $372(230)$ & $\infty$ & $\infty 2 e 6$ & $0 / 5$ & $\overline{\mathrm{PM}}$ & $1338(301)$ & $\infty$ & $\infty 2 e 6$ & $0 / 5$ \\
\hline DE & $3949(1128)$ & $\infty$ & $\infty 2 e 6$ & $0 / 5$ & $\mathrm{DE}$ & $699(258)$ & $\infty$ & $\infty 2 e 6$ & $0 / 5$ & $\mathrm{DE}$ & $3072(370)$ & 8.1 e6 $(9 \mathrm{e} 6)$ & $\infty 2 e 6$ & $0 / 5$ \\
\hline RS-5 & $\mathbf{1}(0)$ & $\infty$ & $\infty 2 e 7$ & $0 / 5$ & RS-5 & $\mathbf{1}(0)$ & $\infty$ & $\infty 2 e 7$ & $0 / 5$ & RS-5 & $1(0)$ & $\infty$ & $\infty 2 e 7$ & $0 / 5$ \\
\hline f9 & & & & 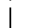 & f28 & & & & & $\mathrm{f} 46$ & & & & \\
\hline$\overline{\mathrm{PM}}$ & $1280(161)$ & $\infty$ & $\infty 2 e 6$ & $0 / 5$ & $\overline{\mathrm{PM}}$ & $937(292)$ & $\infty$ & $\infty 2 e 6$ & $0 / 5$ & $\overline{\mathrm{PM}}$ & $1048(225)$ & $\infty$ & $\infty 2 e 6$ & $0 / 5$ \\
\hline $\mathrm{DE}$ & $2719(146)$ & $\infty$ & $\infty 2 e 6$ & $0 / 5$ & $\mathrm{DE}$ & $1844(675)$ & $5.1 \mathrm{e} 5(1 \mathrm{e} 6)$ & $\infty 2 e 6$ & $0 / 5$ & $\mathrm{DE}$ & $2365(622)$ & $\infty$ & $\infty 2 e 6$ & $0 / 5$ \\
\hline RS-5 & $1(0)$ & $\infty$ & $\infty 2 e^{7}$ & $0 / 5$ & $\mathrm{RS}-5$ & $\mathbf{1}(0)$ & $\infty$ & $\infty 2 e^{7}$ & $0 / 5$ & $\mathrm{RS}-5$ & $1(0)$ & $\infty$ & $\infty 2 e 7$ & $0 / 5$ \\
\hline f10 & & & & 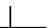 & f29 & & & & 1 & $\mathrm{f} 47$ & & & & 1 \\
\hline$\overline{\mathrm{PM}}$ & \begin{tabular}{|l|}
$782(94)$ \\
\end{tabular} & $\infty$ & $\infty 2 e 6$ & $0 / 5$ & $\overline{\mathrm{PM}}$ & \begin{tabular}{|l}
$972(95)$ \\
\end{tabular} & $\infty$ & $\infty 2 e 6$ & $0 / 5$ & $\overline{\mathrm{PM}}$ & $2327(496)$ & $\infty$ & $\infty 2 e 6$ & $0 / 5$ \\
\hline $\mathrm{DE}$ & $1899(286)$ & $1.4 \mathrm{e} 6(3 \mathrm{e} 6)$ & $\infty 2 e 6$ & $0 / 5$ & $\mathrm{DE}$ & $1923(286)$ & $\infty$ & $\infty 2 e 6$ & $0 / 5$ & $\mathrm{DE}$ & $4671(459)$ & $\infty$ & $\infty 2 e 6$ & $0 / 5$ \\
\hline RS-5 & $\mathbf{1}(0)$ & $\infty$ & $\infty 2 e 7$ & $0 / 5$ & RS-5 & $\mathbf{1}(0)$ & $\infty$ & $\infty 2 e^{7}$ & $0 / 5$ & RS-5 & $1.2(0.5)$ & $\infty$ & $\infty 2 e 7$ & $0 / 5$ \\
\hline f11 & & & & 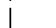 & f3o & & & & 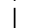 & f48 & & & & . \\
\hline$\overline{\mathrm{PM}}$ & $1(0)$ & $\infty$ & $\infty 2 e 6$ & $0 / 5$ & $\overline{\mathrm{PM}}$ & $2791(586)$ & $8.1 \mathrm{e} 6(1 \mathrm{e} 7)$ & $\infty 2 e 6$ & $0 / 5$ & $\overline{\mathrm{PM}}$ & $866(312)$ & $\infty$ & $\infty 2 e 6$ & $0 / 5$ \\
\hline $\mathrm{DE}$ & $1(0)$ & $\infty$ & $\infty 2 e 6$ & $0 / 5$ & $\mathrm{DE}$ & $4765(702)$ & $\infty$ & $\infty 2 e 6$ & $0 / 5$ & $\mathrm{DE}$ & $1994(868)$ & $\infty$ & $\infty 2 e 6$ & $0 / 5$ \\
\hline RS-5 & $1(0)$ & $8.6 e 7(6 e 7)$ & $\infty 2 e 7$ & $0 / 5$ & RS-5 & $\mathbf{1}(0)$ & $\infty$ & $\infty 2 e 7$ & $0 / 5$ & RS-5 & $1(0)$ & $\infty$ & $\infty 2 e 7$ & $0 / 5$ \\
\hline f12 & & & & j & f31 & & & & i & f 49 & & & & j \\
\hline$\overline{\mathrm{PM}}$ & $4.0(6)$ & $8.8 \mathrm{e} 4(2 \mathrm{e} 5)$ & $\infty 2 e 6$ & $0 / 5$ & $\overline{\mathrm{PM}}$ & $1724(252)$ & $\infty$ & $\infty 2 e 6$ & $0 / 5$ & $\overline{\mathrm{PM}}$ & $1085(272)$ & $\infty$ & $\infty 2 e 6$ & $0 / 5$ \\
\hline $\mathrm{DE}$ & $4.0(3)$ & $1.3 e 4(5150)$ & $\infty 2 e 6$ & $0 / 5$ & $\mathrm{DE}$ & $3566(524)$ & $\infty$ & $\infty 2 e 6$ & $0 / 5$ & $\mathrm{DE}$ & $2230(307)$ & $\infty$ & $\infty 2 e 6$ & $0 / 5$ \\
\hline RS-5 & $\mathbf{1}(0)$ & $\infty$ & $\infty 2 e 7$ & $0 / 5$ & $\mathrm{RS}-5$ & $1(0)$ & $\infty$ & $\infty 2 e^{7}$ & $0 / 5$ & $\mathrm{RS}-5$ & $1(0)$ & $\infty$ & $\infty 2 e 7$ & $0 / 5$ \\
\hline f13 & & & & i & f32 & & & & 1 & f5o & & & & 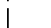 \\
\hline$\overline{\mathrm{PM}}$ & $46(65)$ & $1.8 \mathrm{e} 6(2 \mathrm{e} 6)$ & $\infty 2 e 6$ & $0 / 5$ & $\overline{\mathrm{PM}}$ & $2759(775)$ & $\infty$ & $\infty 2 e 6$ & $0 / 5$ & $\overline{\mathrm{PM}}$ & $2732(606)$ & $\infty$ & $\infty 2 e 6$ & $0 / 5$ \\
\hline $\mathrm{DE}$ & $47(66)$ & $8.0 \mathrm{e} 6(1 \mathrm{e} 7)$ & $\infty 2 e 6$ & $0 / 5$ & $\mathrm{DE}$ & $5052(295)$ & $\infty$ & $\infty 2 e 6$ & $0 / 5$ & $\mathrm{DE}$ & $7753(6868)$ & $\infty$ & $\infty 2 e 6$ & $0 / 5$ \\
\hline RS-5 & $1(0)$ & $\infty$ & $\infty 2 e^{7}$ & $0 / 5$ & $\mathrm{RS}-5$ & $613(2)$ & $\infty$ & $\infty 2 e^{7}$ & $0 / 5$ & $\mathrm{RS}-5$ & $1.4(1)$ & $\infty$ & $\infty 2 e 7$ & $0 / 5$ \\
\hline f14 & & & & & f33 & & & & & f51 & & & & i \\
\hline$\overline{\mathrm{PM}}$ & $6.2(5)$ & $\infty$ & $\infty 2 e 6$ & $0 / 5$ & $\overline{\mathrm{PM}}$ & $885(468)$ & $3.8 \mathrm{e} 4(6 \mathrm{e} 4)$ & $\infty 2 e 6$ & $0 / 5$ & $\overline{\mathrm{PM}}$ & $2149(378)$ & $\infty$ & $\infty 2 e 6$ & $0 / 5$ \\
\hline $\mathrm{DE}$ & $6.2(6)$ & $\infty$ & $\infty 2 e 6$ & $0 / 5$ & $\mathrm{DE}$ & $1972(585)$ & $8622(510)$ & $\infty 2 e 6$ & $0 / 5$ & $\mathrm{DE}$ & $3970(295)$ & $\infty$ & $\infty 2 e 6$ & $0 / 5$ \\
\hline RS-5 & $1(0)$ & $\infty$ & $\infty 2 e 7$ & $0 / 5$ & RS-5 & $\mathbf{1}(0)$ & $\infty$ & $\infty 2 e 7$ & $0 / 5$ & RS-5 & $6.0(2)$ & $\infty$ & $\infty 2 e 7$ & $10 / 5$ \\
\hline f15 & & & & 1 & f34 & & & & & f52 & & & & L \\
\hline$\overline{\mathrm{PM}}$ & \begin{tabular}{|l}
$921(426)$ \\
\end{tabular} & $\infty$ & $\infty 2 e 6$ & $0 / 5$ & $\overline{\mathrm{PM}}$ & $1855(334)$ & $\infty$ & $\infty 2 e 6$ & $0 / 5$ & $\overline{\mathrm{PM}}$ & $2094(69)$ & $\infty$ & $\infty 2 e 6$ & $0 / 5$ \\
\hline $\mathrm{DE}$ & $1627(814)$ & $\infty$ & $\infty 2 e 6$ & $0 / 5$ & $\mathrm{DE}$ & $3213(190)$ & $\infty$ & $\infty 2 e 6$ & $0 / 5$ & $\mathrm{DE}$ & $3596(364)$ & $\infty$ & $\infty 2 e 6$ & $0 / 5$ \\
\hline RS-5 & $\mathbf{1}(0)$ & $\infty$ & $\infty 2 e 7$ & $0 / 5$ & RS-5 & $\mathbf{1}(0)$ & $\infty$ & $\infty 2 e^{7}$ & $0 / 5$ & RS-5 & $1(0)$ & $\infty$ & $\infty 2 e 7$ & $0 / 5$ \\
\hline f16 & & & & i & f35 & & & & I & f53 & & & & | \\
\hline$\overline{\mathrm{PM}}$ & $346(133)$ & $\infty$ & $\infty 2 e 6$ & $0 / 5$ & $\overline{\mathrm{PM}}$ & $1(0)$ & $\infty$ & $\infty 2 e 6$ & $0 / 5$ & $\overline{\mathrm{PM}}$ & $394(445)$ & $4.7 \mathrm{e} 5(6 \mathrm{e} 5)$ & $\infty 2 e 6$ & $0 / 5$ \\
\hline $\mathrm{DE}$ & $516(152)$ & $\infty$ & $\infty 2 e 6$ & $0 / 5$ & $\mathrm{DE}$ & $1(0)$ & $\infty$ & $\infty 2 e 6$ & $0 / 5$ & $\mathrm{DE}$ & $762(1201)$ & $6821(89)$ & $\infty 2 e 6$ & $0 / 5$ \\
\hline RS-5 & $1(0)$ & $\infty$ & $\infty 2 e 7$ & $0 / 5$ & RS-5 & $1(0)$ & $\infty$ & $\infty 2 e 7$ & $0 / 5$ & RS-5 & $1(0)$ & $\infty$ & $\infty 2 e 7$ & $0 / 5$ \\
\hline f17 & & & & & f36 & & & & & f54 & & & & \\
\hline$\overline{\mathrm{PM}}$ & $1083(354)$ & $\infty$ & $\infty 2 e 6$ & $0 / 5$ & $\overline{\mathrm{PM}}$ & $113(157)$ & $\infty$ & $\infty 2 e 6$ & $0 / 5$ & $\overline{\mathrm{PM}}$ & $1615(375)$ & $\infty$ & $\infty 2 e 6$ & $0 / 5$ \\
\hline $\mathrm{DE}$ & 1816(978) & $\infty$ & $\infty 2 e 6$ & $0 / 5$ & $\mathrm{DE}$ & $177(172)$ & $8.0 e 6(7 \mathrm{e} 6)$ & $\infty 2 e 6$ & $0 / 5$ & $\mathrm{DE}$ & $3204(339)$ & $\infty$ & $\infty 2 e 6$ & $0 / 5$ \\
\hline RS-5 & $1.8(1)$ & $\infty$ & $\infty 2 e 7$ & $0 / 5$ & RS-5 & $1(0)$ & $\infty$ & $\infty 2 e 7$ & $0 / 5$ & RS-5 & $1(0)$ & $\infty$ & $\infty 2 e 7$ & $0 / 5$ \\
\hline f18 & & & & & f37 & & & & & f55 & & & & \\
\hline$\overline{\mathrm{PM}}$ & $1(0)$ & $\infty$ & $\infty 2 e 6$ & $0 / 5$ & $\overline{\mathrm{PM}}$ & $133(88)$ & $\infty$ & $\infty 2 e 6$ & $0 / 5$ & $\overline{\mathrm{PM}}$ & $771(116)$ & $\infty$ & $\infty 2 e 6$ & $0 / 5$ \\
\hline DE & $1(0)$ & $8.0 e 6(1 \mathrm{e} 7)$ & $\infty 2 e 6$ & $0 / 5$ & $\mathrm{DE}$ & $186(160)$ & $\infty$ & $\infty 2 e 6$ & $0 / 5$ & $\mathrm{DE}$ & $1905(232)$ & $\infty$ & $\infty 2 e 6$ & $0 / 5$ \\
\hline RS-5 & $1(0)$ & $\infty$ & $\infty 2 e 7$ & $0 / 5$ & RS-5 & $\mathbf{1}(0)$ & $\infty$ & $\infty 2 e 7$ & $0 / 5$ & RS-5 & 1(0) & $\infty$ & $\infty 2 e 7$ & $0 / 5$ \\
\hline f19 & & & & & & & & & & & & & & \\
\hline$\overline{\mathrm{PM}}$ & \begin{tabular}{|l|}
$768(116)$ \\
\end{tabular} & $\infty$ & $\infty 2 e 6$ & $0 / 5$ & & & & & & & & & & \\
\hline $\mathrm{DE}$ & $1427(652)$ & $\infty$ & $\infty 2 e 6$ & $0 / 5$ & & & & & & & & & & \\
\hline RS-5 & $1(0)$ & $\infty$ & $\infty 2 e 7$ & $0 / 5$ & & & & & & & & & & \\
\hline
\end{tabular}

Table 3: Average runtime (aRT) to reach given targets, measured in number of function evaluations, in dimension 20. For each function, the aRT and, in braces as dispersion measure, the half difference between 10 and 90\%-tile of (bootstrapped) runtimes is shown for the different target $\Delta I$-values as shown in the top row. \#succ is the number of trials that reached the last target $I^{\text {ref }}+10^{-5}$. The median number of conducted function evaluations is additionally given in italics, if the target in the last column was never reached. Entries, succeeded by a star, are statistically significantly better (according to the rank-sum test) when compared to all other algorithms of the table, with $p=0.05$ or $p=10^{-k}$ when the number $k$ following the star is larger than 1, with Bonferroni correction of 110 . Best results are printed in bold. 